\title{
Comparative Finite Element Analysis of Jaipur Foot and Polyurethane Foot
}

\author{
Priya Sharma, Shubhda Sharma, S. Vidhya, M. K. Mathur \\ Centre for Biomedical Research, VIT University Vellore, Tamilnadu Jaipur, India \\ Email: priya_vit2009@yahoo.in, shubhda.vit@gmail.com, vidhyavalentina@gmail.com,drmk1@yahoo.com
}

Received 2013

\begin{abstract}
FEA is amongst best methods that help users to solve complex problems. There are fixed number of nodes in each element of the model that define the element boundaries to which boundary condition and loads can be applied. The geometry of the structure, the load applications, stress and displacement gradients can be approximated in a accurate manner, if the mesh is finer. The problem with the foot was unusual cracks in JP Foot and early breakage of PU Foot due to crack propagation [1]. To solve this problem we modelled the foot using SolidWorks and performed FEA Analysis for single leg below knee amputee patients. After analysis, it has been concluded that JP Foot as compared to PU Foot has more stress bearing capacity but has less displacement threshold due to its material properties [2]. This work will lead to optimization of both the feet thus enhancing the durability of foot.
\end{abstract}

Keywords: Load Applications; Crack Propagation; Durability; Solid Works; Stress; Displacement

\section{Introduction}

A Finite element analysis usually consists of three principal steps:

1) Pre-processing: The user constructs a model on which analysis needs to be done and its geometry gets divided into multiple number of discrete elements, or sub regions, which forms nodes while connecting at discrete points [3].

2) Analysis: The user selects restraints and proper loads needed to be applied on the model and optimizes the limits for stress, tolerance and factor of safety accordingly.

3) Post processing: Reports with stress and displacement tables as well as plots are generated for a particular loading condition applied on the model. FEA provides a solution by predicting failure of designed model due to unperceived stress by representing problem areas in used material and allowing analyst to analyse all the speculative stresses within. This designing and testing of Foot can be successfully done using SolidWorks 2008 software.

- COSMOSXpress is a simulation technique of solidworks which performs FEA of solid models. It ensures the performance and quality of the design before it reaches to production stage. Comprehensive analysis tools perform digital model testing for insight at early stage in the process of designing [3]. It is suitable for stress analysis of simple parts, providing the ability to simulate effect of force or pressure loads on those parts. Thus it helps in determining various methods to reduce material costs and weight, to improve manufacturability and durability, to optimize boundaries, and finally to compare design alternatives best suited to customer requirements. A 3d model of Foot size 6 was designed using Solid Works and FEA analysis has been performed.

\section{Materials and Methodology}

Materials Required for Jaipur Foot are Microcellular rubber compound, Cosmetic rubber, Cushion rubber, and Tread rubber. Materials Required for Polyurethane Foot are Polyurethane foam made up of (polyol + isocynate) and Polyurethane elastomer [2]. Physical (Density), mechanical (Elastic Modulus, Shear Modulus, Poisson Ratio, Tensile Strength and Yield Strength) and thermal properties (Thermal Expansion and Thermal Conductivity) [2] values were taken for both the foot and according to that the material was finalized. Properties were given as an input to software as material property before analysis.

\subsection{Software Required}

Software used for modelling is SOLID WORKS 2008. Software used for FEA is COSMOSXpress Analysis Wizard (A simulation Technology of Solid Works 2008)

\subsection{Data Collection}

Data collection regarding the weights of the patients suf- 
fering from single leg (below knee) amputee of varying age group (Figure 2) in different gait cycle conditions [4] (mid stance, heel strike, heel off) [5] was done.

\section{Steps of Analysis}

These are the various steps being followed (Figures 1-5) during COS-MOSX press simulation for FEA analysis.

\section{Results and Discussion}

\subsection{Factor of Safety (FOS)}

FOS is the ratio of material strength to design load.

Standard FOS for JP \& PU foot is considered to be 1 (as material strength should always be more then design load in any condition). The data collected for various weights and conditions from below knee single amputee
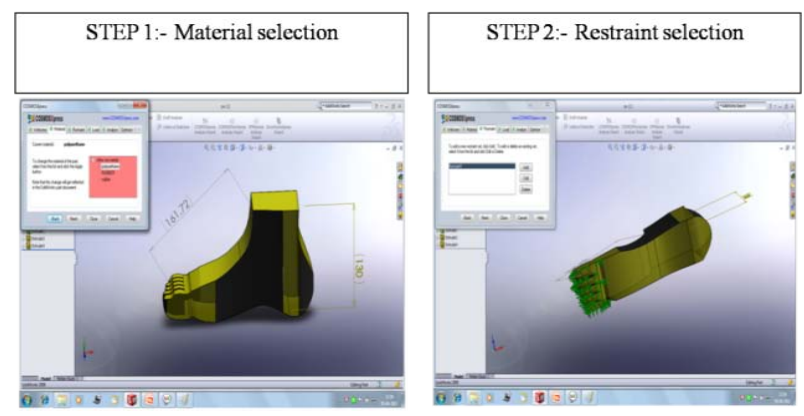

Figure 1. Step 1 and 2 of FEA.
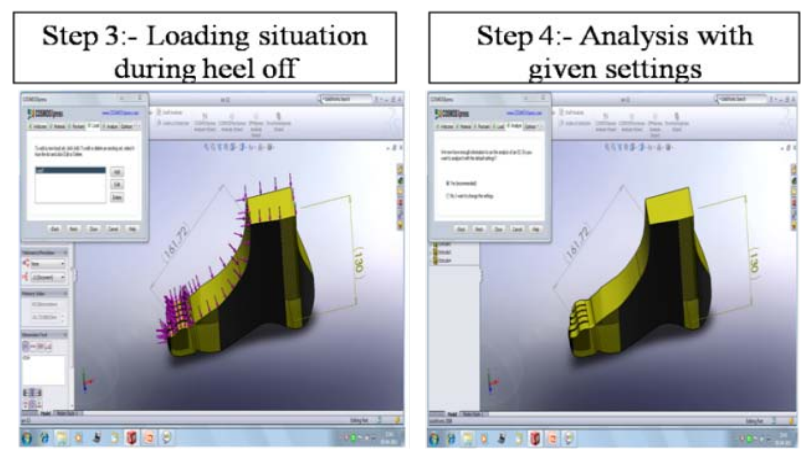

Figure 2. Step 3 and 4 of FEA.
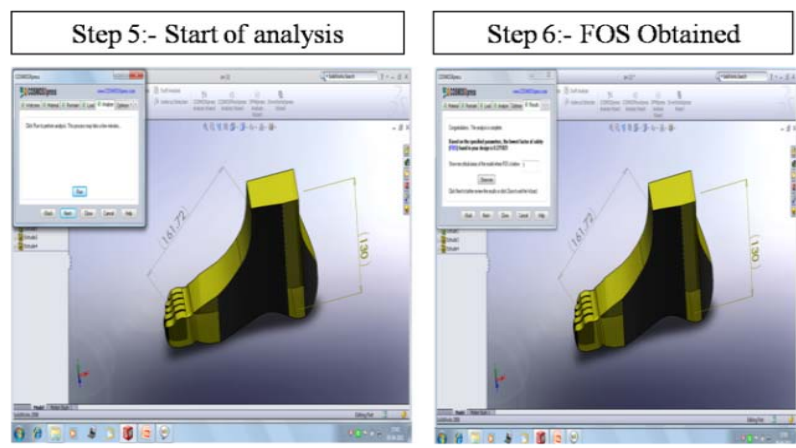

Figure 3. Step 5 and 6 of FEA.
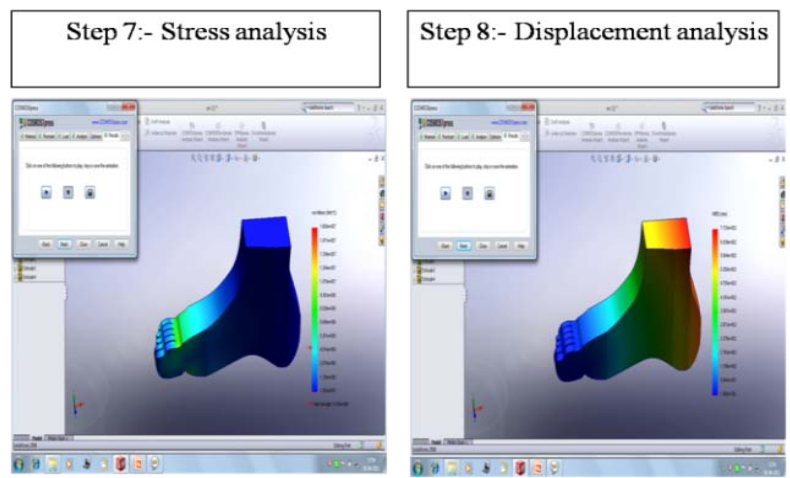

Figure 4. Step 7 and 8 of FEA.
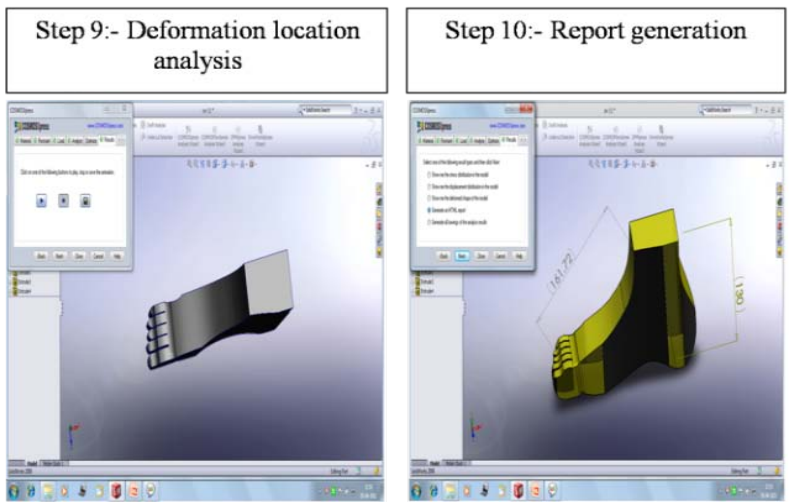

Figure 5. Step 9 and 10 of FEA.

patients of JP \& PU Foot were incorporated in COSMOSXpress Simulation Wizard for the FEA analysis of both the Feet. FOS values obtained for JP Foot are always higher than the FOS values obtained for PU Foot at the given loads in different conditions of gait cycle [6], so it can be concluded that material strength of JP Foot is better than material strength of PU Foot. Thus JP Foot bears a lower risk of failure due to crack propagation.

\subsection{Results of Stress Analysis}

When various loads were applied to the model in different gait cycle conditions [7], maximum \& minimum stress values were obtained for PU \& JP Foot which is shown through graphical representation (Graphs 4.2.1-4.2.5).

According to stress values (max. \& min.) obtained during analysis, it is concluded:

- During midstance \& heel strike maximum stress condition, JP foot is much safer than PU foot as polyurethane being soft material cannot withstand large amount of load. So max. Stress values of PU foot are higher.

- During midstance \& heel strike minimum stress condition, PU foot is much safer than JP foot comparatively as polyurethane is a flexible material and it can absorb a certain amount of shock that occurs at minimum stress condition [8]. 


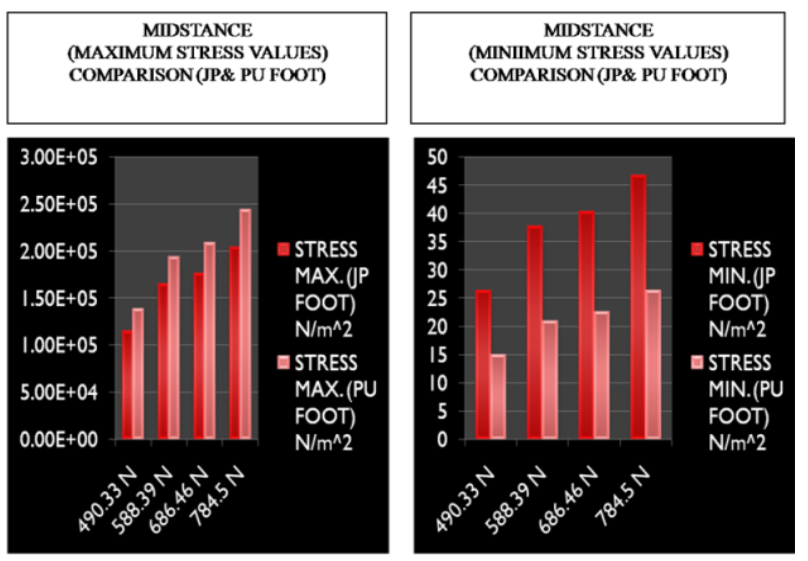

Graph 4.2.1. Comparative stress values for midstance.

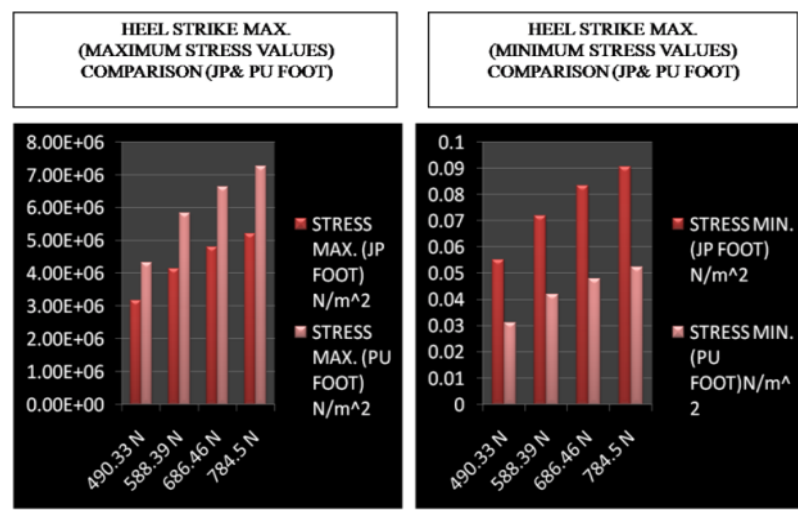

Graph 4.2.2. Comparative stress values for heel strike max.

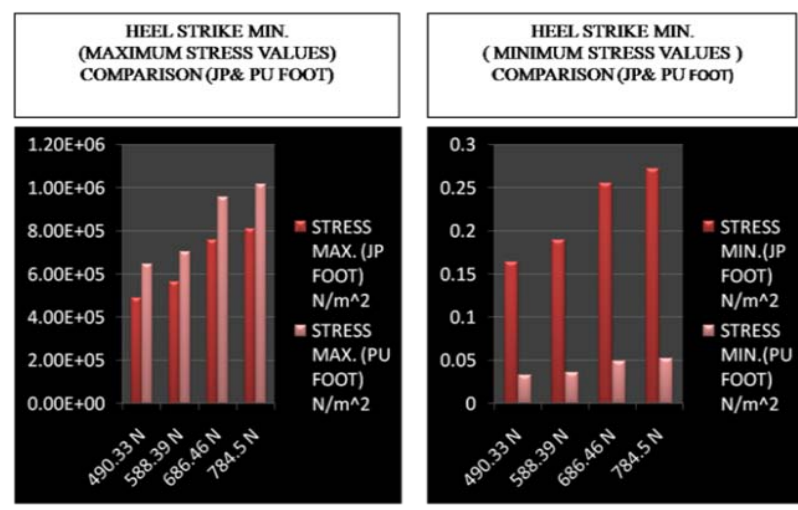

Graph 4.2.3. Comparative stress values for heel strike min.

- During heel off (max. \& min.) stress condition, JP foot is always safer than PU foot as the phalanges \& metatarsals region bear the full load, the threshold stress absorbance level of polyurethane material get exceeded and thus PU foot is vulnerable to early cracks due to stress applied.

\subsection{Results of Displacement Analysis}

When various loads were applied to the model in different gait cycle conditions displacement values were
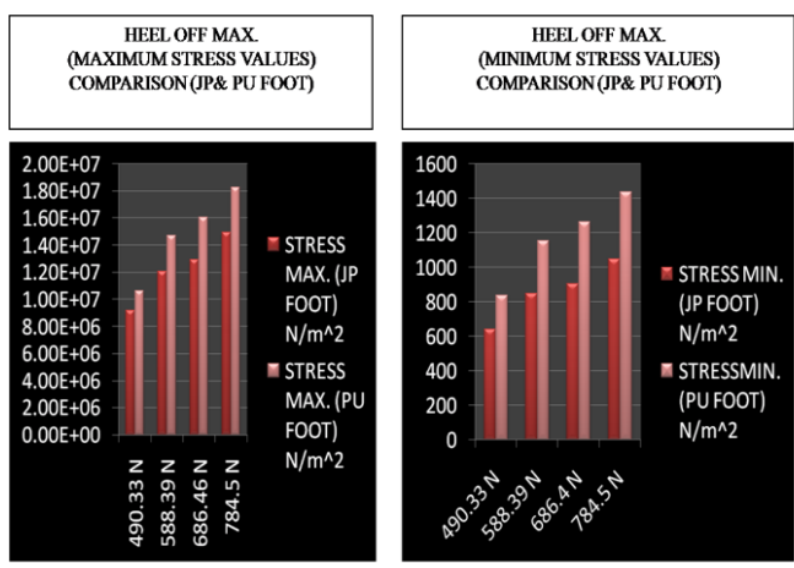

Graph 4.2.4. Comparative stress values for heel off max.
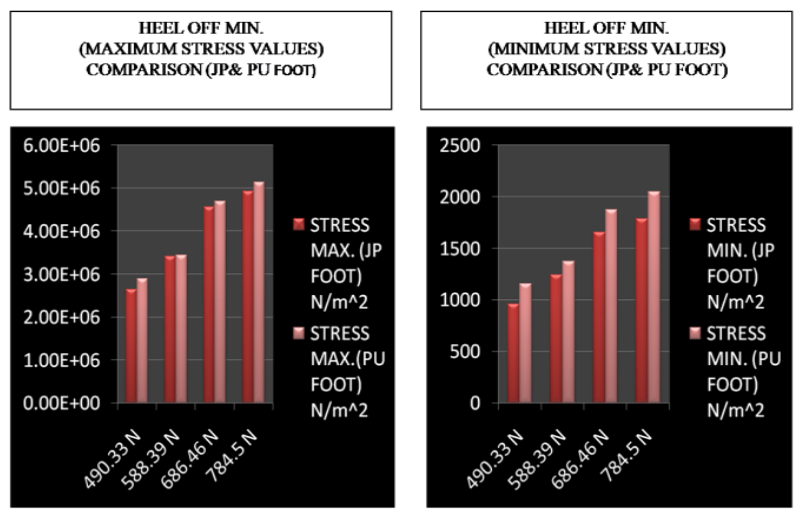

Graph 4.2.5. Comparative stress values for heel off $\mathrm{min}$.

obtained and comparative analysis of displacement has been performed for JP \& PU Foot which is shown through graphical representation (Graphs 4.3.1-4.3.3).

\section{Conclusion and Optimization}

Over the last decade there has been an adequate increment of computer applications in the field of rehabilitation and prosthetic designing. FEA is also one amongst these revolutionary ways to analyze an existing or future model for prediction of its breakage point, durability, stress bearing capacity and displacement. We performed FEA analysis of JP and PU Foot to test the stress bearing capacity, to compare the durability, displacement and crack propagation in both the foot. After analyzing stress and displacement according to their values obtained as well as their location points it is concluded that JP Foot has a better material strength and stress bearing capacity than PU Foot although PU Foot displacement threshold is higher than JP Foot due to its property "resilience" which is very high for polyurethane foam and due to that PU Foot is able to regain its shape back up to a certain extent and displacement appears to be lower than JP Foot. We have done a proper FEA analysis of both the foot without compromising the quality of model and results. 


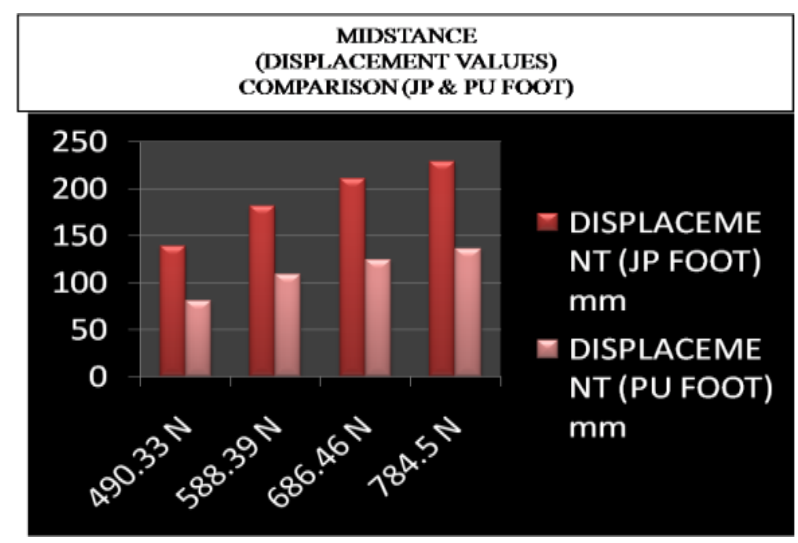

Graph 4.3.1. Comparative displacement values for midstance.
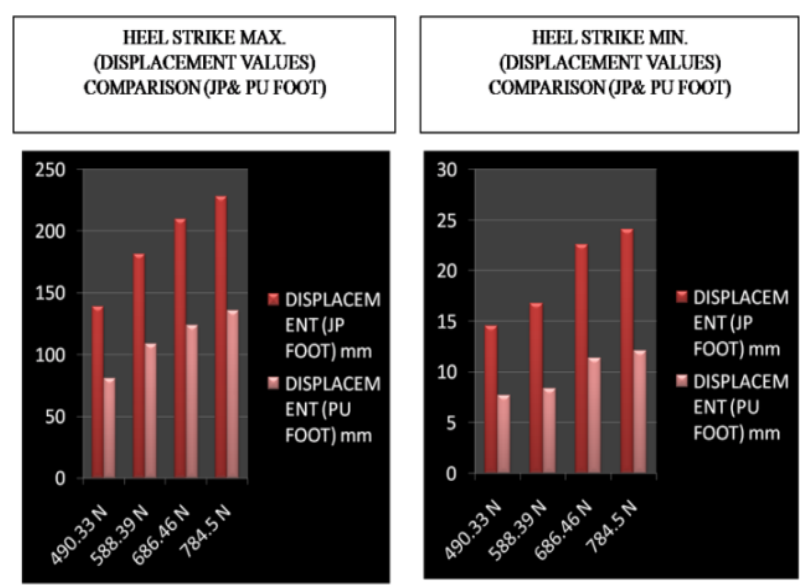

Graph 4.3.2. Comparative displacement values for heel strike max. and min.
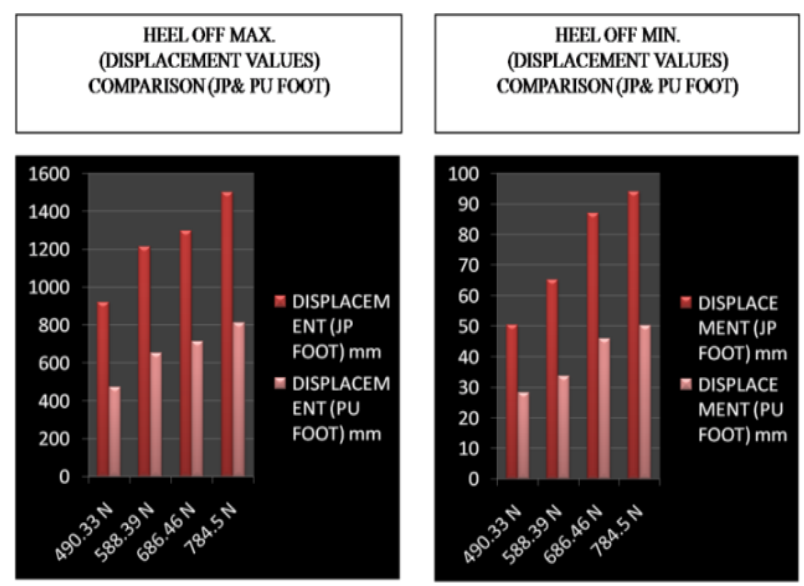

Graph 4.3.3. Comparative displacement values for heel off max. and min.

For better results in future this comparative FEA analysis could be done using Force Plate which can predict the stress and displacement during dynamic gait cycle.
Through the results obtained, design can be optimized in following ways:

- Material strengthening of JP Foot at maximum stress areas by increasing the proportion of cosmetic and cushion rubber to avoid future crack propagation in those areas.

- JP Foot can have less displacement than the present model if it has a layer of PU Foam inside it which will provide an optimal resilience level to the foot along with the usual ratio of other types of rubber compounds which will provide the same strength as the foot have up till now.

- PU Foot can have similar stress bearing capacity as JP Foot if the outer polyurethane elastomeric layer of the foot is prepared separately with the thickness $25 \%$ more than that of present model.

- PU Foot being lighter in weight (approx 30\%) less than JP Foot has a brighter probability of being used in future if the above mentioned modifications are implemented.

\section{REFERENCES}

[1] Bis Jaipur-Foot Final Report, BMVSS, Jaipur.

[2] V. V. Karunakaran, "Quality Assurance and Optimization Studies of Light Weight PU Prosthetic Foot,” Trends in Biomaterials and Artificial Organs, Vol. 19, No. 2, 2006, pp. 63-69.

[3] L. Zheng, et al., “3D Finite Element Analysis of Bone Stress around Distally Osseointegrated Implant for Artificial Limb Attachment,” Key Engineering Material, Vol. 288-289, 2005, pp. 653-656.

[4] D. A. Winter, "Kinematic and Kinetic Patterns in Human GAIT: VARIABILITY and Compensating Effects," $\mathrm{Hu}$ man Movement Science, Vol. 3, 1984, pp. 51-76. http://dx.doi.org/10.1016/0167-9457(84)90005-8

[5] M. M. Rodgers, "Dynamic Foot Biomechanic,” Journal of Orthopaedic \& Sports Physical Therapy, Vol. 6, 1995, pp. 306-316.

http://dx.doi.org/10.2519/jospt.1995.21.6.306

[6] W. C. C. Lee, M. Zhang, P. P. Y. Chan and D. A. Boone, "Gait Analysis of Low-Cost Flexible-Shank Transtibial Prostheses," Neural Systems and Rehabilitation Engineering, Vol. 14, 2006, pp. 370-377. http://dx.doi.org/10.1109/TNSRE.2006.881540

[7] C. W. Chan and A. Rudins, "Foot Biomechanics during Walking and Running," Mayo Clinic proceedings Mayo Clinic, Vol. 69, 1994, pp. 448-461.

[8] M. Argin and G. G. Karady, "Characterization of Polyurethane Foam Dielectric Strength,” Dielectrics and Electrical Insulation, Vol. 14, 2008, pp. 350-356. http://dx.doi.org/10.1109/TDEI.2008.4483452 